\title{
Tracoma: ainda uma importante causa de cegueira
}

\section{"Trachoma: still being an important blinding disease"}

Silvana Artioli Schellini', Roberta Lilian Fernandes de Sousa²

\section{Resumo}

O Tracoma é uma doença milenarmente conhecida e que persiste como causa de cegueira entre nós. As autoras chamam a atenção para os fatores relacionados com a transmissão, apresentam dados históricos e de distribuição da doença no Brasil e no mundo, comentam sobre o agente, os sinais e os sintomas desta conjuntivite crônica. Ainda reforçam a necessidade de capacitar os profissionais para o diagnóstico, o que possibilitará a detecção e tratamento. O reflexo destas atitudes será a contribuição para a eliminação desta importante causa de cegueira do nosso meio.

Descritores: Tracoma/história; Conjuntivite crônica; Cegueira; Brasil

\section{Abstract}

Trachoma is a disease known thousand years ago and still as a potential blindness disease all over the world. The authors call attention to the factors related with the transmission, present historical data and distribution of the disease in Brazil and in the world, comment on the agent, the signs and symptoms of this chronic conjunctivitis. Still, reinforce the need to enable professionals for the diagnosis, detection and treatment. The reflection of these attitudes will be the contribution to the elimination of this important disease as a blindness cause among us.

Keywords: Trachoma/history; Chronic conjunctivitis; Blindness; Brazil

\footnotetext{
"Faculdade de Medicina de Botucatu -Universidade Estadual "Júlio de Mesquita Filho" (UNESP) - Botucatu (SP), Brasil; ${ }^{2}$ Pós-Graduanda do Programa Bases Gerais da Cirurgia - Faculdade de Medicina de Botucatu - Universidade Estadual "Júlio de Mesquita Filho" (UNESP) - Botucatu (SP), Brasil;

Faculdade de Medicina de Botucatu - Universidade Estadual "Júlio de Mesquita Filho" (UNESP) - Botucatu (SP), Brasil.

Os autores declaram inexistir conflitos de interesse
}

Recebido para publicação em 18/3/2011 - Aceito para publicação em 27/6/2011 


\section{INTRODUÇÃO}

A pesar de milenarmente conhecido, o Tracoma figura ainda dentre as doenças que preocupam o mundo, estando presente especialmente nos países em desenvolvimento. No ano de 2003, a Organização Mundial de Saúde (OMS) estimava a existência de 84 milhões de pessoas com Tracoma ativo no mundo, 76 milhões com triquíase tracomatosa e cinco milhões com sérios riscos para a visão e potencial desenvolvimento de cegueira ${ }^{(1)}$.

O Tracoma é considerado pelas organizações internacionais do campo da saúde como uma das "doenças negligenciadas", doença para a qual tem havido pouco ou nenhum investimento da indústria, no desenvolvimento de novas técnicas de diagnóstico, medicamentos e vacinas. Essa doença tem sido negligenciada inclusive pela academia, o que se pode constatar pelo pequeno número de estudos e publicações sobre ela.

A doença está relacionada com baixas condições sócioeconômicas e baixos índices de desenvolvimento humano, sendo descrita em locais com precárias condições de habitação, grande concentração populacional, precariedade de saneamento básico, baixos níveis educacional e cultural. Outros fatores relacionados com a presença do Tracoma seriam: presença de insetos vetores, deslocamentos populacionais, presença de outras doenças oculares, precariedade no abastecimento de água e coleta de lixo e íntima relação com a estrutura social ${ }^{(2,3)}$.

A doença figura entre as causas de cegueira a serem prevenidas, fazendo parte do programa GET 2020 (Global Elimination of Trachoma) da OMS, que visa exterminar as causas preveníveis de cegueira no planeta até $\mathrm{o}$ ano de $2020^{(4)}$.

A OMS tem como meta para o combate desta doença a estratégia "SAFE" (S=cirurgia corretiva de triquíase; $\mathrm{A}=$ antibióticos; $\mathrm{F}=$ limpeza da face; $\mathrm{E}=$ saneamento), baseada em medidas bastante simples e que envolvem higiene pessoal, acompanhada de melhoria das condições de vida ${ }^{(4)}$. Lavar o rosto( ${ }^{(5)} \mathrm{e}$ usar latrinas ${ }^{(6)}$ tem promovido diminuição significativa dos casos em algumas regiões. Interessante pesquisa realizada na Amazônia brasileira mostrou que ter água disponível não é suficiente; é preciso ter hábitos de higiene que possam impedir que a doença seja adquirida e que se propague ${ }^{(7)}$.

Para que haja sucesso na tarefa de eliminar o Tracoma como causa de cegueira, há que se desenvolver estratégias de atuação nas áreas de maior prevalência, como é o Brasil, envolvendo projetos que favoreçam o diagnóstico dos casos agudos e também detectem e tratem as formas cicatriciais.

\section{Distribuição do Tracoma no Brasil e no mundo}

O Tracoma é presente desde os primeiros registros humanos, em diferentes civilizações e momentos, tais como na China (século XXVII a.C.), Suméria (século XXI a.C.), Egito (século XIX a.C.), Grécia (século V a.C.) e Roma (século I a.C.).

O Tracoma se disseminou pelo antigo continente europeu juntamente com as grandes migrações de povos, dentre eles os mongóis que se lançaram em guerras e conquistas por grande extensão territorial. Na Idade Média a doença era abundante no Mundo Islâmico e na Grécia, sendo levado para o restante da Europa, onde se tornou endêmico.

A partir da Europa, foi trazido pela colonização para o Continente Americano. Na segunda metade do século XIX e início do século XX, o Tracoma achava-se amplamente disseminado em todo o mundo. No decorrer do século XX, com a melhoria das condições de vida, consequente à industrialização e ao desenvolvimento econômico, o Tracoma desapareceu da Europa, América do Norte e Japão.

No entanto, continua a ser um importante problema de saúde pública em grande parte dos países subdesenvolvidos, principalmente na África, Oriente Médio, Índia e Sudoeste da Ásia. O Tracoma ainda existe em menores proporções na América Latina e Oceania.

\section{Tracoma no Brasil}

O Tracoma chegou ao Brasil por três vertentes: região do Cariri (Ceará), São Paulo e Rio Grande do Sul. Relata-se que teria sido introduzido no Brasil a partir do século XVIII, no Nordeste, com a deportação dos ciganos que haviam sido expulsos de Portugal e se estabelecido nas Províncias do Ceará e Maranhão, constituindo-se então os primeiros "focos" de Tracoma no país, dos quais o mais famoso foi o "foco do Cariri", no sul do atual estado do Ceará. Além do "foco do Nordeste", outros dois "focos" teriam contribuído decisivamente para a disseminação do Tracoma no país, os "focos" de São Paulo e do Rio Grande do Sul, que teriam se iniciado com a intensificação da imigração européia para esses dois estados, a partir da segunda metade do século XIX. Com a expansão da fronteira agrícola em direção ao oeste, o Tracoma foi disseminando-se e tornou-se endêmico em praticamente todo o Brasil, sendo encontrado hoje em todo o território nacional.

A primeira medida de controle do Tracoma adotada no Brasil foi de iniciativa do Governo do Estado de 
São Paulo, que em 1904 proibiu a entrada de imigrantes com Tracoma no Porto de Santos, a exemplo do que era feito nos Estados Unidos. Esta medida, porém, não teve sucesso, devido à pressão dos fazendeiros de café, que necessitavam da mão de obra imigrante. Desta forma, a proibição foi substituída por uma multa para o dono do navio que trouxesse imigrantes com Tracoma. Em 1906, inicia-se em São Paulo a primeira "Campanha contra o Tracoma" realizada no país, e em 1914 começam a ser instalados, também em São Paulo, os primeiros serviços especializados em Tracoma, os "Postos Antitracomatosos".

Em nível nacional, a primeira medida de controle do Tracoma foi tomada em 1923, quando foi decretado o "Regulamento do Departamento Nacional de Saúde Pública", que proibia o desembarque de imigrantes com Tracoma, medida esta que, naquele momento, já era totalmente inócua, já que o mesmo encontrava-se amplamente disseminado no país, e não mais dependia da imigração para sua manutenção.

A partir de 1938, o estado de São Paulo iniciou a implantação de uma rede de serviços especializados em Tracoma, os "Dispensários do Tracoma", anexos aos Centros de Saúde e localizados nos locais onde a doença era mais prevalente. Esta rede chegou a ter mais de 200 unidades, cobrindo quase a totalidade do estado de São Paulo. Depois de diversas medidas de combate a doença, com postos de detecção ligados aos postos de saúde e também na zona rural, chegou-se a diminuição importante do número de casos.

O Governo Federal iniciou, em 1943, a realização da "Campanha Federal Contra o Tracoma", por iniciativa do Departamento Nacional de Saúde Pública. Esta Campanha foi incorporada ao "Departamento Nacional de Endemias Rurais - DENERu", quando da sua criação em 1956, e posteriormente à SUCAM (Superintendência Nacional de Campanhas de Saúde Pública), criada em 1970.

O ciclo de desenvolvimento econômico iniciado nos anos 50 e que perdura até o "milagre econômico" dos anos 70, teve um profundo impacto na ocorrência do Tracoma no Brasil. Observou-se uma diminuição acentuada no número de casos detectados em todo o país, e chegou-se mesmo a considerar que o Tracoma havia sido erradicado em alguns estados, como em São Paulo, sendo que em 1978, na implantação do Sistema de Vigilância Epidemiológica no Estado de São Paulo, o Tracoma não foi incluído no elenco de doenças de notificação compulsória.

Todo o aparato que havia sido montado para o combate à endemia foi redimensionado e o Tracoma passou a não ser mais pesquisado, nem ensinado nas escolas médicas e nos cursos de especialização. Passa-se a ter outra realidade, ligada ao desconhecimento da afecção.

O mito da "erradicação" do Tracoma, cunhado em meados da década de 70 do século XX, persiste até hoje. A pretensão de pertencer a um mítico "país desenvolvido" e o fetiche tecnológico da incorporação de equipamentos oftalmológicos cada vez mais sofisticados, fez com que os departamentos de Oftalmologia da maioria das universidades "esquecessem" de olhar o seu entorno, não identificando a doença ocular ainda frequente na população.Assim, os especialistas continuaram a propagar o mito da erradicação e muitos de nós, oftalmologistas, fazemos parte de uma geração que nunca recebeu treinamento formal durante o curso de especialização em Oftalmologia para detecção e manejo da doença.

Na década de 80 do século passado, o Tracoma volta a ter destaque, a partir do encontro de Tracoma na cidade de Bebedouro, estado de São Paulo, nas diversas fases, denotando que a doença nunca havia sido eliminada naquele município ${ }^{(8)}$.

As ações de vigilância epidemiológica do Tracoma foram retomadas pela Secretaria de Estado da Saúde de São Paulo, detectando a endemia em mais de 150 municípios do estado, alguns com altos coeficientes de prevalência e com complicações e sequelas ${ }^{(9)}$.

Em 1990, as atividades de controle do Tracoma no âmbito nacional passaram a fazer parte das atribuições da Fundação Nacional de Saúde - FNS. O Ministério da Saúde vem mantendo as ações de controle nas regiões com maior prevalência, através da FNS, estando o controle da doença na Gerência Técnica Nacional de Endemias Focais.

Usando sistemática investigativa, o Tracoma foi detectado em várias regiões do Brasil ${ }^{(9-18)}$. Entretanto, a ideia da erradicação do Tracoma ainda permanece como dominante entre a comunidade científica brasileira até os dias atuais.

Pesquisas epidemiológicas feitas em todo o Brasil reforçam a existência do Tracoma na realidade de todos os municípios nos quais foi feita a investigação, com taxas de detecção variáveis: Alagoas (4,5\%); Rio Grande do Norte $(3,61 \%)$, Bahia (3,58\%), Paraíba $(3,81 \%)$, Roraima $(4,34 \%)$, São Paulo $(4,11 \%)$, Rio Grande do Sul (4,60\%), Tocantins (5,33\%), Sergipe (5,84\%), Paraná (6,2\%), Espírito Santo (4,74\%), Ceará $(7,81 \%)$, Acre $(8,34 \%)$, São Paulo - SP $(2,2 \%)$, Santa Catarina (4,9\% e Joinville), Manaus (4,8\%), Rio de Janeiro $(8,78 \% \text { em Duque de Caxias) })^{(3)}$. Mesmo em regiões onde não era reportada a doença, estudos bem con- 
duzidos mostraram que a mesma está presente, inclusive com altas taxas de detecção, o que levou os pesquisadores a concluir que é importante divulgar que o Tracoma não foi erradicado e deve permanecer no diagnóstico diferencial de conjuntivite folicular crônica em crianças e adolescentes em alguns municípios ${ }^{(19)}$. Estudos feitos no interior de São Paulo também apontam a presença da doença em diferentes taxas: Presidente Prudente - SP (2,6\%), Franco da Rocha - SP (1,5\%), Francisco Morato - SP $(3,6 \%)^{(14,19,20)}$.

Assim é possível observar que, apesar da diminuição acentuada na prevalência e na incidência do Tracoma, a doença sempre existiu em nosso meio, acometendo majoritariamente as populações mais carentes e desassistidas, presente inclusive nas grandes metrópoles.

\section{O agente}

O Tracoma é causado pela C. trachomatis, que possui 15 sorotipos diferentes já identificados. Os sorotipos L1, L2 e L3 são os grandes responsáveis pela síndrome do linfogranuloma venéreo; A, B, Ba e C são mais frequentemente associados ao tracoma; os de $\mathrm{D}$ a K estão ligados a outras manifestações sexualmente transmitidas, sendo que os sorotipos mais frequentes são os do tipo D, E e F. Assim como em outras doenças sexualmente transmissíveis (DST), a infecção genital é uma porta de entrada e aumenta o risco de contrair o vírus HIV, agente da síndrome da imunodeficiência adquirida (Aids/SIDA).

O controle do tracoma depende da identificação de portadores assintomáticos, os quais representam um reservatório para o agente patogênico. Acredita-se que a imunidade é apenas parcialmente protetora, pois o risco de desenvolver sequelas como gravidez ectópica ou infertilidade aumenta com os sucessivos episódios de infecção.

\section{Os sinais, os sintomas e o diagnóstico}

$\mathrm{O}$ agente provoca uma conjuntivite crônica, que se acompanha de poucos sinais e sintomas, dentre eles: prurido ocular, hiperemia leve, pouca ou nenhuma secreção ocular. A dificuldade maior para o diagnóstico decorre exatamente da cronicidade, o que faz com que os sintomas sejam frustros ou ausentes. Confunde-se, ainda, com a conjuntivite alérgica que não infrequentemente se encontra associada ao quadro da conjuntivite tracomatosa. O diagnóstico do Tracoma é essencialmente clínico. A confirmação laboratorial deve ser utilizada, para a constatação da presença do agente etiológico na comunidade, e não para a confirmação de cada caso.
A OMS orienta que o diagnóstico de Tracoma deve ser dado quando houver pelo menos dois dos seguintes sinais clínicos: folículos na conjuntiva tarsal superior, folículos no limbo ou fossetas de Herbert, cicatriz conjuntival típica e pannus no limbo superior ${ }^{(21,22)}$.

Visando a pesquisa da doença em comunidades (trabalhos de campo), a mesma organização estabeleceu um esquema simples de classificação do Tracoma, com a finalidade de simplicidade diagnóstica e implicações epidemiológicas (Tabela 1). Cada um dos sinais tem importância para compreensão do estado epidemiológico da população(23).

A prevalência da doença ativa é representada pela proporção de pessoas com TF (tracoma folicular) e TI (tracoma folicular intenso); aqueles com TI representam indivíduos com doença grave, requerendo pronto tratamento. A prevalência de TT (triquíase tracomatosa), por sua vez, indica a necessidade de tratamento cirúrgico para aquela comunidade, enquanto que a prevalência de CO (opacidade de córnea) indica o impacto do Tracoma como causa de perda visual.

Há várias formas de pesquisar o Tracoma na população. Atingir a população portadora é mais simples se a pesquisa se inicia pelos escolares, já que as crianças estão reunidas nos espaços didáticos. A partir dos casos positivos, os adultos acometidos (comunicantes) podem ser identificados e todos devem receber o tratamento clínico que atualmente é feito com a administração via oral de Azitromicina. Todos os acometidos passam por exames periódicos para certificação da cura.

Caso a doença tenha permanecido por muitos anos na comunidade investigada, as seguidas reinfecções poderão ocasionar a triquíase tracomatosa, a qual poderá ser responsável por lesões corneanas por traumatismo direto, seguindo-se de opacidade da córnea e perda da visão. A forma cicatricial da doença poderá requerer tratamento cirúrgico.

Esta é a sistemática para quem se lança a campo para pesquisar esta afecção, devendo ser a equipe preparada para tal.

\section{Importância de se pesquisar e ensinar o Tracoma}

Detectar e tratar o Tracoma é muito importante, já que estas medidas são capazes de alterar o quadro da doença. O Tracoma pesquisado em escolares de Botucatu no ano de 1992 mostrou prevalência de $11 \%$, tendo sido os casos e comunicantes tratados ${ }^{(9)}$. Nova pesquisa feita no ano de 2005, conduzida nos mesmos moldes que a anterior, apontou significativa queda do número de casos em relação ao levantamento anterior, com prevalência de TF de $3,7 \%{ }^{(24)}$. 
Pesquisa realizada na cidade de Bauru mostrou interessante faceta da doença com relação ao meio ambiente. Através de geoprocessamento, a distribuição espacial das residências dos alunos afetados foi feita, tendo sido demonstrado que a localização dos casos traduz condições de detecção de outras afecções. Ou seja, o Tracoma pode funcionar como marcador para doenças relacionadas com a pobreza, indicando onde as secretarias de saúde devem investir para a prevenção de doenças ${ }^{(18,25,26)}$. Além disso, há que se pesquisar nas áreas onde a doença está presente, quais seriam os fatores que mantém a doença ativa.

Apesar da importância do Tracoma em todo o território nacional, já demonstrada pelas inúmeras pesquisas que revelaram as taxas de prevalência, o ensino da doença, com raras exceções, não é feito de forma regular nas escolas de Medicina e nos cursos que formam oftalmologistas. É preciso incluir este assunto nos currículos médicos e nos cursos de residência médica, a fim de capacitar pessoas a diagnosticar e tratar adequadamente esta afecção, o que vai contribuir para a erradicação da mesma.

No ano de 2010, o Ministério da Saúde, em ação conjunta com o Ministério da Educação, lançou um Programa de Ensino Tutorial (PET) em Vigilância Epidemiológica. A Faculdade de Medicina de Botucatu teve a aprovação do PET-Tracoma, do qual participa uma equipe multidisciplinar, constituída por membros da Secretaria de Saúde do Município e da Faculdade de Medicina de Botucatu, que estão trabalhando como tutores, no ensino da doença para a comunidade universitária, replicando e criando novos investigadores em Tracoma.

É importante frisar que, mesmo em cidades em que a doença é combatida com busca ativa e sistemática, e tratamento dos casos e comunicantes, a doença ainda se faz presente, e a ampliação do número de profissionais integrados na luta contra a doença é necessária para que se consiga cumprir a meta de extermínio do Tracoma até o ano de 2020, como é o desejo da OMS.

\section{Conclusão}

As autoras chamam a atenção para o Tracoma, uma doença muito antiga, ainda presente em nosso meio e que é potencial causa de cegueira. Comentam sobre a necessidade de se oferecer treinamento para jovens profissionais, a fim de capacitá-los a realizar o diagnóstico clínico desta afecção, o que vai possibilitar o tratamento e afastar o risco de cegueira.

\section{RefERÊNCIAS}

1. World Health Organization. Report of the 2nd Global Scientific Meeting on Trachoma. Geneva, 25-27 August, 2003. Geneva: WHO; 2003.

2. D'Amaral RKK, Cardoso MRA, Medina NH, Cunha ICKO, Waldman EA. Fatores associados ao tracoma em área hipoendêmica da Região Sudeste, Brasil. Cad Saúde Pública. 2005;21(6):1701-8.

3. Lopes MFC. Tracoma: situação epidemiológica no Brasil [dissertação]. Salvador: Instituto de Saúde Coletiva. Universidade Federal da Bahia; 2008.

4. Bailey R, Lietman T. The SAFE strategy for the elimination of trachoma by 2020: will it work? Bull World Health Organ. 2001;79(3):233-6

5. West S, Muñoz B, Lynch M, Kayongoya A, Chilangwa Z, Mmbaga BB, Taylor HR. Impact of face-washing on trachoma in Kongwa, Tanzania. Lancet. 1995;345(8943):155-8.

6. Emerson PM, Lindsay SW, Walraven GE, Dibba SM, Lowe KO, Bailey RL. The Flies and Eyes project: design and methods of a cluster-randomised intervention study to confirm the importance of flies as trachoma vectors in the Gambia and to test a sustainable method of fly control using pit latrines. Ophthalmic Epidemiol. 2002;9(2):105-17.

7. Gonçalves FOR. Distribuição espacial e prevalência do tracoma no município de São Gabriel da Cachoeira, AM [tese]. Ribeirão Preto: Faculdade de Medicina de Ribeirão Preto da Universidade de São Paulo; 2003.

8. Luna EJ, Medina NH, Oliveira MB, de Barros OM, Vranjac A, Melles HH, et al. Epidemiology of trachoma in Bebedouro State of São Paulo, Brazil: prevalence and risk factors. Int J Epidemiol. 1992;21(1):169-77.

9. Medina NH, Gattás VL, Anjos GL, Montuori C, Gentil RM. Prevalência de tracoma em pré-escolares e escolares em Botucatu, São Paulo, Brasil, 1992. Cad Saúde Pública. 2002;18(6):1537-41.

10. Scarpi MJ, Plut RCA, Arruda HO. Prevalência do tracoma no povoado de Mocambo, Estado do Ceará, Brasil. Arq Bras Oftalmol. 1989;52(5):177-9.

11. Scarpi MJ, Silva RJM, Ferreira IA, Barbosa FAC, Plut RCA. Prevalência de tracoma em bairro do município de Palmares, Estado de Pernambuco, Brasil. Arq Bras Oftalmol. 1990;53(4):171-4

12. Scarpi MJ, Gentil R. Sinais e sintomas do tracoma em povoado do Estado da Bahia, Brasil. Arq Bras Oftalmol. 1990;53(6):276-8.

13. Nóbrega MJ, Bonomo PPO, Scarpi MJ, Campos CEG, Juliano Y, Novo NF. Prevalência de tracoma em crianças pré-escolares e escolares da periferia da cidade de Joinvillle, Estado de Santa Catarina, Brasil. Arq Bras Oftalmol. 1993;56(1):13-7.

14. Lucena AR, Cruz AAV, Cavalcanti R. Estudo epidemiológico do tracoma em comunidade da Chapada do Araripe Pernambuco - Brasil. Arq Bras Oftalmol. 2004;67(2):197-200.

15. Alves AP, Medina NH, Cruz AA. Trachoma and ethnic diversity in the Upper Rio Negro Basin of Amazonas State, Brazil. Ophthalmic Epidemiol. 2002;9(1):29-34.

16. Reis ACPP, Chaves C, Cohen JM, Belfort F, Oliveira NPO, Belfort Júnior R. Detecção de tracoma e doenças corneanas em índios da região do Alto Rio Negro. Arq Bras Oftalmol. 2002;65(1):79-81

17. Paula JS, Medina NH, Cruz AAV. Trachoma among the Yanomami Indians. Braz J Med Biol Res. 2002;35(10):1153-7. 
18. Ferraz LCB. Tracoma em criança do ensino fundamental no município de Bauru - Estado de São Paulo, Brasil [tese]. Botucatu: Faculdade de Medicina da Universidade Estadual Paulista; 2006.

19. Damasceno RWF, Santos RR, Cavalcanti TRT, Hida RY, Santos MJ, Santos AMC, Dantas PEC. Tracoma: estudo epidemiológico de escolares em Alagoas - Brasil. Arq Bras Oftalmol. 2009;72(3):355-9.

20. Couto Júnior AS, Scarpi MJ, Guidugli T. Prevalência de tracoma em pré-escolares e escolares no Município de Duque de Caxias - RJ. Rev Bras Oftalmol. 1997;56(7):515-21.

21. Krachmer JH, Mannis MJ, Holland EJ. Chlamydial infections. In: Krachmer JH, Mannis MJ, Holland EJ, editors. Cornea and external diseases: clinical diagnosis and management. St. Louis: Mosby; 1997. Vol 2. p. 780-3.

22. Dawson C. Chlamydial infections. In: Kaufman HE, Baron BA, McDonald MB. The cornea. 2nd ed. Oxford: ButterworthHeinemann; 1998. V. 1. p. 315-30.

23. Thylefors B, Dawson CR, Jones BR, West SK, Taylor HR. A simple system for the assessment of trachoma and its complications. Bull World Health Organ. 1987;65(4):477-83.

24. Schellini SA, Ferraz LB, Hirai FE, Barroso F, Medina NH, Padovani CR. Trachoma in school children in Botucatu, Sao Paulo State, Brazil: prevalence and spatial localization of detected cases. Vision Pan-America. 2008;7(4):110-3.
25. Macharelli CA. Aspectos epidemiológicos do tracoma em crianças do ensino fundamental do município de Bauru - SP: a utilização do geoprocessamento na priorização de recursos do setor saúde [tese]. Botucatu: Faculdade de Medicina da Universidade Estadual Paulista; 2010.

26. Schellini SA, Ferraz LB, Macharelli CA, Opromolla P, Padovani CR. Trachoma in a Community: Georeference as a tool to optimization of resources from the the public health system. In: XXI ISGEO Congress 3rd4thJune 2010, Berlin, Germany.

\section{Endereço para correspondência:}

Silvana A Schellini

Depto de OFT/ORL/CCP

Faculdade de Medicina de Botucatu (UNESP)

CEP 18618-970 - Botucatu (SP), Brasil

Tel/Fax: (14)3811-6256

E-mail: sartioli@fmb.unesp.br 